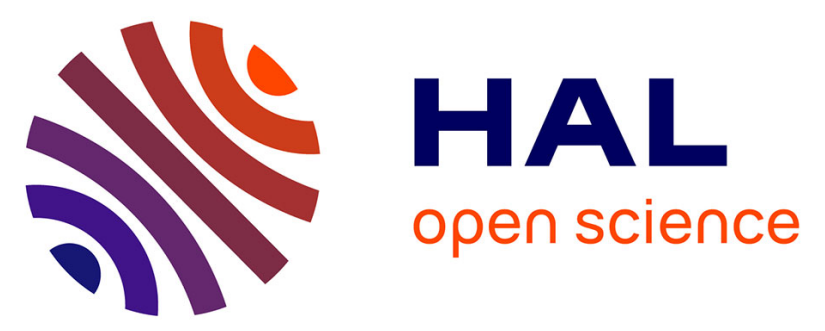

\title{
Membrane targeting of protein tyrosine phosphatase PTPL1 through its FERM domain via binding to phosphatidylinositol 4,5-biphosphate.
}

Guillaume Bompard, Marianne Martin, Christian Roy, Françoise Vignon, Gilles Gf Freiss

\section{To cite this version:}

Guillaume Bompard, Marianne Martin, Christian Roy, Françoise Vignon, Gilles Gf Freiss. Membrane targeting of protein tyrosine phosphatase PTPL1 through its FERM domain via binding to phosphatidylinositol 4,5-biphosphate.. Journal of Cell Science, 2003, 116 (Pt 12), pp.2519-30. $10.1242 /$ jcs.00448 . inserm-00150999

\section{HAL Id: inserm-00150999 https://www.hal.inserm.fr/inserm-00150999}

Submitted on 19 Nov 2009

HAL is a multi-disciplinary open access archive for the deposit and dissemination of scientific research documents, whether they are published or not. The documents may come from teaching and research institutions in France or abroad, or from public or private research centers.
L'archive ouverte pluridisciplinaire HAL, est destinée au dépôt et à la diffusion de documents scientifiques de niveau recherche, publiés ou non, émanant des établissements d'enseignement et de recherche français ou étrangers, des laboratoires publics ou privés. 


\section{Membrane targeting of protein tyrosine phosphatase PTPL1 \\ through its FERM domain via binding to phosphatidylinositol \\ 4,5-biphosphate}

Key words: Protein tyrosine phosphatase, FERM domain, $\mathrm{PI}(4,5) \mathrm{P}_{2}$-binding sites, neomycin, apical localization.

Guillaume Bompard ${ }^{1, *}$, Marianne Martin $^{2}$, Christian Roy ${ }^{2}$, Françoise Vignon ${ }^{1}$ and Gilles Freiss $^{1}$.

${ }^{1}$ Inserm U540, Endocrinologie Moléculaire et Cellulaire des Cancers, Montpellier, France and

${ }^{2}$ Dynamique Moléculaire des Interactions Membranaires, Université Montpellier II, Unité Mixte de Recherche (UMR) CNRS 5539, Montpellier Cedex 5, France.

*Author for correspondence (present address: School of Biosciences, University of Birmingham, UK. email: G.Bompard@bham.ac.uk)

Short title: Tyrosine phosphatase PTPL1 targeting regulation 


\section{Summary}

PTPL1 is the largest known cytoplasmic protein tyrosine phosphatase (PTP) containing a FERM (Four point-1, Ezrin, Radixin and Moesin) domain. Enzyme localization, as well as PTP-substrate specificity, are thought to play a crucial role in the regulation of their activity which determines their functions. Here we report that PTPL1 is predominantly localized at the apical face of plasma membrane enriched in dorsal microvilli once expressed in HeLa cells. By comparing localization of the full-length enzyme versus its FERM domain or FERMdeleted PTPL1 construct, we first conclude that PTPL1-FERM domain is necessary and sufficient to address the wild-type enzyme at the membrane. Two potential phosphatidylinositol 4,5-biphosphate $\left(\mathrm{PI}(4,5) \mathrm{P}_{2}\right)$-binding motifs were identified within PTPL1-FERM sequence. We further showed that mutation of both sites altered PTPL1 localization similarly to FERM domain deletion, and impaired its subcellular distribution as confirmed biochemically by cell-fractionation experiments. Using protein-lipid overlays, we demonstrated an interaction of the FERM domain of PTPL1 with PI(4,5) $\mathrm{P}_{2}$, which was lost after mutation of potential $\mathrm{PI}(4,5) \mathrm{P}_{2}$-binding motifs. Moreover, neomycin which masks $\mathrm{PI}(4,5) \mathrm{P}_{2}$ polar-heads was shown to decrease by $50 \%$ the association of PTPL1 with cytoskeletal fraction. These results identify the crucial role of the FERM domain in PTPL1 intracellular targeting and demonstrate that localization of PTPL1 is regulated by phosphoinositide metabolism. 


\section{Introduction.}

Many cellular processes such as cell growth, differentiation, locomotion, cell-cell interactions are controlled by tyrosyl phosphorylation of specific cellular proteins. Reversible phosphorylation on tyrosine residue depends on a balance between protein tyrosine kinase (PTK) and protein tyrosine phosphatase (PTP) activities (for a review, see Neel and Tonks, 1997). Since any misregulation can lead to severe disease, these activities must be tightly regulated (Hunter, 1997; Zhang, 2001). Relatively little is known about the function and regulation of PTPs. Like for PTKs, the activity of transmembrane PTPs can, in some instances, be regulated by an inter- or intra-molecular mechanism (eg., Wallace et al., 1998). Concerning cytosolic PTPs, targeting to highly restricted subcellular micro-compartments is thought to play an important role in the control of their activity and substrate specificity. However, for some PTPs, it can be achieved in combination with an intrinsic property carried by their catalytic domain allowing specific substrate recognition (for review, Tonks and Neel, 2001). Cytosolic PTPs possess a remarkable variety of non-catalytic domains. Among their structural motifs, some were shown to target the enzyme towards its correct intracellular context ('zip code' model, see Mauro and Dixon, 1994; Fischer, 1999).

PTPL1/FAP-1/hPTP1E/PTP-BAS, is the largest known cytoplasmic PTP (250 kDa) and belongs to a family of PTPs characterized by the presence of a FERM (Four point-1, Ezrin, Radixin and Moesin) domain (Saras et al., 1994; Sato et al., 1995; Banville et al., 1994; Maekawa et al., 1994). This family comprises PTPH1 (Yang and Tonks, 1991), PTPMEG (Gu et al., 1991), PTP36/PTPD2/Pez (Sawada et al., 1994; Moller et al., 1994; Smith et al., 1995), PTPD1/PTP-RL10 (Moller et al., 1994; Higashitsuji et al., 1995). PTPL1 presents no characteristic feature in its far $\mathrm{N}$-terminal end except for a potential leucine zipper followed in the protein core by a FERM domain, five PDZ domains and the C-terminal catalytic domain. 
FERM domains are commonly found within a family of peripheral membrane proteins that mediate linkage of the cytoskeleton to the plasma membrane (for review, Chishti et al., 1998; Bretscher, 1999; Mangeat at al., 1999). This family comprises the erythrocyte protein 4.1, ezrin, radixin, moesin, merlin (product of Nf2, a tumor suppressor) and talin. The FERM domain has been well characterized in ERM proteins, prototypes of membrane-cytoskeleton linkers. ERMs harbor a major actin binding site within their C-terminus and a FERM domain at their N-terminal part which binds a number of proteins (EBP 50/NHE3-RF, RhoGDI,...) some of which are transmembrane proteins such as CD44, CD43, ICAM-2, ICAM-3. Furthermore, ERM proteins can self-associate in an intra- or inter-molecular manner through homotypic and heterotypic interaction between their $\mathrm{N}$ - and C-terminal ends masking several sites involved in the binding of membrane proteins and actin. Such interactions are regulated by tyrosine or threonine phosphorylation but also by phosphatidylinositol 4,5-biphosphate $\left(\mathrm{PI}(4,5) \mathrm{P}_{2}\right)$ (for review, Bretscher, 1999). Finally, ezrin localization was recently shown to be altered by mutation of $\mathrm{PI}(4,5) \mathrm{P}_{2}$-binding motifs found in its FERM domain (Barret et al, 2000).

We previously showed that the expression of PTPL1/FAP-1 was specifically induced by antiestrogens, like hydroxytamoxifen (OH-Tam), in estrogen receptor positive human breast cancer cells (Freiss et al., 1998). This induction was correlated with an increase of PTP activity associated with the insoluble fraction (membrane and cytoskeleton) and was involved in the early steps of anti-growth factor action of OH-Tam (Freiss and Vignon, 1994; Bompard et al., 2002).

In the present study, we investigated PTPL1 localization and evaluated the role of its FERM domain in order to understand how enzyme activity could be regulated. We showed that the FERM domain is necessary and sufficient to target PTPL1 to the apical cell membrane and to allow it to be enriched in dorsal microvilli in HeLa cells. Analysis of PTPL1-FERM sequence 
suggested the presence of two potential $\mathrm{PI}(4,5) \mathrm{P}_{2}$-binding sites. We showed that these two sites, involved in phosphatidylinositol phosphate lipid (PIP) binding in general, and $\mathrm{PI}(4,5) \mathrm{P}_{2}$ in particular, play a major role in PTPL1 cellular localization and association with the cytoskeletal fraction.

Collectively, our data bring the first indication for the importance of $\mathrm{PI}(4,5) \mathrm{P}_{2}$ binding through the FERM domain for the intracellular targeting and association with cytoskeleton of the human protein tyrosine phosphatase PTPL1. 


\section{Materials and Methods}

\section{Materials}

Enzymes were purchased from Life Technologies, Inc., Eurogentec, New England Biolabs and Promega. Monoclonal antibodies against epitope HA (12CA5) and epitope VSV-G (P5D4) were purchased from Roche. Anti-vimentin monoclonal (V9) was from Immunotech. Anti-actin (A 2066) and phalloidin-TRITC polyclonals were from Sigma. Anti-tubulin monoclonal was a gift from Dr. N. Lamb (Montpellier).

\section{Construction of expression plasmids}

All proteins encoded from pHM6 vector (Roche) are HA-tagged at their N-terminus and 6Histagged at their C-terminus. All constructs were verified by sequencing.

The expression construct pHM6-PTPL1 encoding influenza virus hemagglutinin (HA)epitope tagged PTPL1 (HA-PTPL1) was created as follows. Oligonucleotides carrying Bsu15I and Psp5II restriction sites were introduced in SacII-NotI sites of pHM6 vector. The cDNA encoding the N-terminal part of PTPL1 was cloned by PCR using pSV7d-PTPL1 (gift of Prof. C.H. Heldin) as a template in SacII-Bsu15I sites of modified pHM6 vector. SacII site was introduced by PCR in frame with the second coding codon (removal of start codon) and Bsu15I was an endogenous PTPL1 site. By the same approach, the cDNA encoding the C-terminal part of PTPL1 was then inserted in Psp5II-NotI sites of modified pHM6 vector. Psp5II was an endogenous PTPL1 site and NotI site was introduced by PCR in order to remove PTPL1 stop codon. Finally, the remaining PTPL1 cDNA from pSV7d-PTPL1 was inserted in Bsu15I-Psp5II sites generating pHM6-PTPL1 (aa 2-2459, Fig. 1).

pHM6-FERM encoding HA-tagged FERM (aa 511-958) domain of PTPL1 (HA-FERM) was cloned by PCR in EcoRI-SacII of pHM6 (Fig.1). pHM6-PTPL1 $\triangle$ FERM (del aa 523-909, 
Fig.1) was generated as followed. pBSK-FERM (aa 511-958) was digested by BamHI and MscI, and the open reading frame was restored by introducing double-stranded oligonucleotides (hybridized oligonucleotides) generating pBSK- $\triangle \mathrm{FERM}$. $\triangle \mathrm{FERM}$ was then excised by StuI/PacI digestions and the fragment was cloned in pBSK-PTPL1 (aa 2-2459) in order to generate pBSK-PTPL1 $\triangle$ FERM. Full length PTPL1 $\triangle$ FERM was then subcloned in pHM6 through SacII/NotI sites. Mutagenesis of potential $\mathrm{PI}(4,5) \mathrm{P}_{2}$-binding sites was performed with oligonucleotides using QuickChange ${ }^{\mathrm{TM}}$ Site-Directed Mutagenesis Kit (Stratagene).

The changed bases are underlined and only sequences of the forward oligonucleotides are given. The oligonucleotide (5'-3') used for introducing K645,646,647N mutations (KN1 mutant) in pHM6-FERM was GGAAAGAAGAACCAAAㅁA드AAㅁACCAAAGCCACTG (16 cycles, $\left.95^{\circ} \mathrm{C}, 30 \mathrm{~s} ; 55^{\circ} \mathrm{C}, 1 \mathrm{~min} ; 68^{\circ} \mathrm{C}, 15 \mathrm{~min}\right) . \mathrm{K} 824,825,830,831,832 \mathrm{~N}$ mutations (KN2 mutant) were introduced in two times. First, the following oligonucleotide was used to mutate

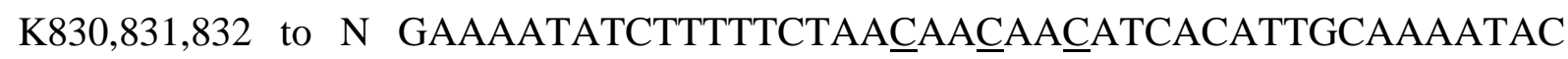
(same conditions described above) generating an intermediate mutant. This intermediate mutant was used as template to introduce $\mathrm{K} 824,825 \mathrm{~N}$ mutation with the following oligonucleotide CCATGGAGGGAAACCAACEAAC̣ATATCTTTTTCTAACAAC (same conditions described above) generating KN2 mutant. KN1-2 mutant was generated using oligonucleotide carrying K645,646,647N mutations described above and using pHM6-FERM KN2 as template. pHM6-PTPL1 KN1-2 construct was generated using the same approach as described below for pHM6-PTPL1 $\triangle$ FERM.

The in vitro expression construct pRSET-FERM encoding 6His-tagged FERM domain (519958) was created by subcloning BamHI-HindIII fragment from pHM6-FERM in pRSET vector (Invitrogen). pRSET-FERM KN1, KN2 and KN1-2 were created by the same approach 
using respective BamHI-HindIII fragments from pHM6-FERM-KN1, pHM6-FERM-KN2 and pHM6-FERM-KN1-2.

pCB6 Nt-Ezrin (aa 1-310) expressing VSV-G epitope tagged FERM domain of ezrin was a generous gift from Dr. M. Arpin (Paris).

\section{Cell culture}

HeLa and COS1 cells were cultured in Dulbecco's Modified Eagle's medium (DMEM) supplemented with $10 \%$ fetal calf serum (FCS), $2.5 \mathrm{U} / \mathrm{ml}$ penicillin and $2.5 \mu \mathrm{g} / \mathrm{ml}$ streptomycin (Invitrogen). Cells were transfected either with the calcium phosphate precipitation method or using LipofectAMINE PLUS ${ }^{\mathrm{TM}}$ reagents (Invitrogen) according to the manufacturer.

\section{Cell fractionation}

COS 1 cells were seeded 24 hours before transfection at a density of 125,000 cells per $35-\mathrm{mm}$ dish. As indicated, cells were treated with $10 \mathrm{mM}$ neomycin (Sigma) for 24 hours. Forty hours after transfection, cells were washed two times with ice cold PBS and lysed in fractionation buffer (10 mM PIPES, pH 6.8, $250 \mathrm{mM}$ sucrose, $3 \mathrm{mM} \mathrm{MgCl} 2,120 \mathrm{mM} \mathrm{KCl,} 1$ mM EGTA, $0.15 \%$ Triton X-100, 1 $\mathrm{mM} \mathrm{Na}_{3} \mathrm{VO}_{4}, 25 \mathrm{mM} \mathrm{NaF}, 5 \mathrm{mM}$ sodium pyrophosphate, $1 \mathrm{mM}$ PMSF and 0.02-0.04 trypsin inhibitor unit (TIU) of aprotinin) for $5 \mathrm{~min}$ at $4^{\circ} \mathrm{C}$. The total lysate was centrifuged at $25,000 \mathrm{x}$ g for $15 \mathrm{~min}$ at $4^{\circ} \mathrm{C}$ yielding a supernatant containing depolymerised tubulin and monomeric actin, and a pellet containing polymerised cytoskeletons (microfilaments and intermediate filaments) resuspended in fractionation buffer (volume comparable to supernatant) and sonicated. 


\section{Immunoblotting analysis}

Equal volumes of each fraction were separated on SDS/polyacrylamide gel and electrotransferred onto PVDF membrane. Blots were then stained with Coomassie blue (R250, Sigma) to ensure that protein amounts were comparable. Membranes were blocked with TBST (50 mM Tris, $\mathrm{pH} 7.5,150 \mathrm{mM} \mathrm{NaCl}, 0.1 \%$ Tween 20 ) containing $5 \%$ non fat-milk and immunoblotted with anti-HA, anti-actin, anti-vimentin and anti-tubulin monoclonal or polyclonal antibodies. Primary antibodies were detected using horseradish peroxydaseconjugated goat anti-mouse IgG or anti-rabbit IgG, and Western Lighting (PerkinElmer Life Sciences, Inc.).

\section{Immunofluorescence study}

Twenty hours after transfection, cells were fixed in medium with $3.7 \%$ formaldehyde for 20 min and permeabilized in TBS buffer (50 mM Tris, $\mathrm{pH} 7.5,150 \mathrm{mM} \mathrm{NaCl})$ containing $0.2 \%$ Triton X-100 for $4 \mathrm{~min}$. Cells were incubated with anti-HA mAb for $60 \mathrm{~min}$ at room temperature, washed and incubated with FITC-conjugated anti-mouse Ig for $30 \mathrm{~min}$ at room temperature. After final wash and mounting in Gel/Mount (Biomeda), cells were examined with a laser scanning confocal microscope (Bio-Rad Laboratories) using a 63x1.4 oil immersion objective or a DMR A microscope PL APO 63x oil immersion objective (1.32 NA) with appropriate filters (Leica) and images were recorded with a cooled CCD Micromax camera (1,300 x 1,030 pixels, RS; Princeton Instruments Inc.).

\section{Protein-lipid overlays}

${ }^{35}$ S-labeled PTPL1-FERM domain and its derived mutants, generated by the TnT in vitro transcription-coupled translation system (Promega) from pRSET-FERM vector, were incubated with Ni-NTA agarose column (Qiagen) for 1 hour at room temperature. After two 
washes with $\mathrm{S}$ buffer $\left(50 \mathrm{mM} \mathrm{NaH} \mathrm{PO}_{4}, 20 \mathrm{mM}\right.$ Tris, $300 \mathrm{mM} \mathrm{NaCl}, 5 \mathrm{mM}$ imidazole, $1 \mathrm{mM}$ PMSF and 0.02-0.04 trypsin inhibitor unit (TIU) of aprotinin, $\mathrm{pH} 8)$ and $\mathrm{W}$ buffer $(20 \mathrm{mM}$ Tris, $150 \mathrm{mM} \mathrm{NaCl}, 30 \mathrm{mM}$ imidazole), recombinants proteins were eluted in the $\mathrm{E}$ buffer (20 $\mathrm{mM}$ Tris, $100 \mathrm{mM} \mathrm{NaCl}, 300 \mathrm{mM}$ imidazole). 40,000 cpm of ${ }^{35} \mathrm{~S}$-labeled PTPL1-FERM domain and its derived mutants were incubated for 2 hours at room temperature in $5 \mathrm{ml}$ TBST, 3\% BSA with PIP-Strips (Echelon Biosciences Inc.) pre-saturated with TBST, 3\% BSA. After three washes with TBST, binding of FERM domain was quantified with a Fuji BAS1000 Bioimaging Analyzer (Raytest). 


\section{$\underline{\text { Results }}$}

The FERM domain of PTPL1 is necessary and sufficient for apical localization of the enzyme

FERM domains are thought to direct proteins at the interface between cell membrane and cytoskeleton. We investigated the localization of PTPL1 and the role of its FERM domain in HeLa epithelial cells by expressing various HA-epitope tagged constructs (Fig.1 A) which sizes had been controlled to be correct by SDS-PAGE (Fig 1 B, C). Upon transient expression in HeLa cells, PTPL1 was found almost exclusively enriched apically at the plasma membrane and little was present in the cytoplasm (Fig. 2, A). In addition, PTPL1 staining presented a colocalization with F-actin in microvilli and plasma membrane (Fig. 2, C). In the case of strong overexpression, PTPL1 was found to induce apical bleb-like structure where it colocalized with F-actin (data not shown). We evaluated the specific role of the FERM domain in two ways by expressing constructs encoding either only the FERM domain (HAFERM) or the full-length enzyme deleted of the FERM domain (HA-PTPL1 $\triangle$ FERM). The FERM domain, similarly to the full-length PTPL1, localized to the apical cell membrane and was enriched in microvilli (Fig. 2, D-F). Furthermore, the FERM domain also induced cell extensions (Fig. 2, D) as observed with the ezrin FERM domain (Martin et al., 1997). By contrast, PTPL1 $\triangle$ FERM was found mainly cytosolic with few accumulation at the plasma membrane or in microvilli (Fig. 2, G-I). These results clearly demonstrate that the FERM domain is necessary and sufficient for apical localization of PTPL1.

\section{Mutation of two potential PI(4,5) $\mathbf{P}_{2}$ binding sites altered PTPL1 localization}

FERM domains of ERM family proteins are known to bind transmembrane proteins such CD44, CD43, ICAM-1, -2, -3 and also PI(4,5) $\mathrm{P}_{2}$ (for review Bretscher, 1999; Mangeat at al., 1999). Furthermore, ERM-CD44 binding is enhanced, in vitro, by $\mathrm{PI}(4,5) \mathrm{P}_{2}$ (Hirao, 1996). 
Recently, it has been shown that mutation of $\mathrm{PI}(4,5) \mathrm{P}_{2}$-binding motifs in the FERM domain of ezrin altered its intracellular localization (Barret and al., 2000). Sequence analysis of the PTPL1-FERM domain revealed the presence of two potential PI(4,5) $\mathrm{P}_{2}$-binding sites (Fig. 3, bold bars). $\mathrm{PI}(4,5) \mathrm{P}_{2}$ binding motifs are characterised by cluster of basic amino acids; e.g., $\mathrm{KKXXXXXX(K/R)K}$ in FERM domains of ERM. The first potential PI(4,5) $\mathrm{P}_{2}$-binding motif found in PTPL1-FERM domain, KEEPKKK, was located at amino acids 641-647 and the second, KKISFSKKK, at amino acids 824-832 (Fig. 3). In order to evaluate the role of such motifs in PTPL1 localization, several mutations of the lysine residues located in the presumed sites were introduced in FERM domain. Lysines 645, 646 and 647 in the first site and lysines $824,825,830,831,832$ in the second site were mutated to asparagines generating respectively $\mathrm{KN} 1$ and $\mathrm{KN} 2$ mutants. KN1-2 mutant was obtained by combining both sets of mutations.

As for HeLa cells, when expressed in COS1 fibroblasts PTPL1-FERM domain localized in apical microvilli and was able to induce membrane extensions in many cells (Fig. 4, A). Such effect has also been reported with the ezrin FERM domain (Martin et al., 1997) and has been shown to be lost by preventing $\mathrm{PI}(4,5) \mathrm{P}_{2}$ binding (Barret et al., 2000). Based on this result, we evaluated the effect of mutations of potential $\mathrm{PI}(4,5) \mathrm{P}_{2}$-binding sites, within the PTPL1FERM domain, on its cellular localization (cell extensions, membrane and microvilli). KN1 and KN2 mutants, partly inhibited cell extension processes but had few effects on the localization of the FERM domain at the membrane and microvilli (Fig. 4, B, C). However combination of both sets of mutations, strongly altered the localization of the FERM domain and KN1-2 mutant presented in most cells a diffuse cytosolic staining (Fig. 4, D). It is important to note that KN2 mutant presented also a localization comparable to KN1-2 mutant in some cells (data not shown). Thus, both potential $\mathrm{PI}(4,5) \mathrm{P}_{2}$-binding sites seemed to cooperate for proper intracellular targeting of the FERM domain of PTPL1. 
Since the most drastic effect on FERM domain localization was obtained by mutating both potential $\mathrm{PI}(4,5) \mathrm{P}_{2}$-binding motifs, KN1-2 mutations were secondarily introduced in the fulllength enzyme to evaluate their roles on its localization in HeLa cells. As shown with COS1 cells, KN1-2 mutations abrogated membrane and microvilli localization of FERM domain as well as cell extensions (compare Fig. 2, D with Fig. 5, A). Similarly, HA-epitope tagged PTPL1 KN1-2 localization was strongly altered compared to the wild-type protein, presenting a diffuse cytosolic staining around the nucleus (compare Fig. 2, A with Fig. 5, D). Such a mislocalization was comparable to that observed for the PTPL1 $\triangle$ FERM mutant (Fig. 2, G). Similar effects were obtained in COS1 cells (data not shown). In summary, these results demonstrated that the two potential $\mathrm{PI}(4,5) \mathrm{P}_{2}$-binding sites found within the PTPL1-FERM domain are crucial for FERM and PTPL1 localization. It finally suggested that PTPL1 localization could be regulated by phosphoinositide metabolism.

\section{PTPL1-FERM domain binds $\mathrm{PI}(4,5) \mathrm{P}_{2}$ in vitro}

To directly assess the PI(4,5) $\mathrm{P}_{2}$ binding property of the FERM domain of PTPL1, we carried out protein-lipid overlays. In vitro labeled transcribed and translated 6His-tagged PTPL1-FERM domains (wt, KN1, KN2 and KN1-2) were used to probe nitrocellulose membrane containing various immobilized phosphoinositides (PIPs), after their purification on and elution from nickel-agarose columns. Figure 6A shows such eluted proteins separated on SDS-PAGE and visualized by autoradiography. Using this assay, 6His-FERM domain was found to specifically bind 4' and/or 5' phosphorylated PIPs, ie $\mathrm{PI}(4) \mathrm{P}, \mathrm{PI}(5) \mathrm{P}, \mathrm{PI}(3,4) \mathrm{P}_{2}$, $\mathrm{PI}(3,5) \mathrm{P}_{2}, \mathrm{PI}(4,5) \mathrm{P}_{2}$ and $\mathrm{PI}(3,4,5) \mathrm{P}_{3}$ (Fig. 6, B). A very weak interaction with $\mathrm{PI}(3) \mathrm{P}$ was sometimes observed whereas no interaction was detected with non phosphorylated phosphatidylinositol and other control phospholipids (PA, PS, LPA, LPC, PE, PE, S1P). KN1 mutation slightly affects PIPs binding of the 6His-FERM domain (Fig. 6, B). This effect was 
more pronounced with KN2 mutation (Fig. 6, B). However, KN1-2 mutation abolishes the binding of 6His-FERM domain to immobilized PIPs by $70 \%$ to $80 \%$ (Fig. 6, B).

This result clearly demonstrates that the FERM domain of PTPL1 directly binds PI(4,5) $\mathrm{P}_{2}$ in vitro through the two binding sites located between amino-acids 641-647 and 824-832.

\section{Subcellular distribution of PTPL1 correlates with PI $(4,5) P_{2}$ binding.}

Having established that the FERM domain of PTPL1 binds PI(4,5) $\mathrm{P}_{2}$ in vitro, the subcellular distributions of wild-type, KN1, KN2, KN1-2 HA-tagged FERM domains, as well as wildtype, KN1-2, $\triangle$ FERM HA-tagged PTPL1 were compared, upon cell fractionation of COS1 cells, in order to provide biochemical evidence for the differences in localization observed by immunofluorescence. Following cell fractionation, wild-type FERM domain was found to be strongly associated with the Triton $\mathrm{X}-100$ insoluble fraction containing polymerised actin (F-actin) and vimentin (Fig. 7, A). KN1 mutant displayed a comparable distribution even if the amount present in the soluble fraction, containing depolymerised tubulin and actin (Gactin), was more significant than that observed with the wild-type FERM (Fig. 7, A). By contrast, KN2 and KN1-2 mutants were only found to be associated with the soluble fraction (Fig. 7, A) in accordance with the poor affinity of these proteins for $\mathrm{PI}(4,5) \mathrm{P}_{2}$ (Fig. 6, B). Following the same approach, effects of KN1-2 mutations and FERM deletion on full-length PTPL1 subcellular distribution were analysed. As for its FERM domain, wild-type PTPL1 showed a marked association with the cytoskeletal fraction that is abolished in KN1-2 or $\triangle$ FERM mutants (Fig. 7, B). $35 \%$ of total HA-PTPL1 was associated with Triton X-100 insoluble fraction versus $3.6 \%$ and $1.9 \%$ for HA-PTPL1 KN1-2 and HA-PTPL1 $\triangle$ FERM respectively (Fig. 8). The subcellular distribution of full-length PTPL1 did not require its catalytic activity since the localization of an inactive mutant (C2389S) was indistinguishable from that of the native enzyme (data not shown). Our data demonstrated that association of 
PTPL1 with Triton X-100 insoluble fraction depended on its FERM domain and on the integrity of at least the second $\mathrm{PI}(4,5) \mathrm{P}_{2}$-binding site.

To confirm that the association of the wild-type FERM domain and PTPL1 with the particulate fraction was due to $\mathrm{PI}(4,5) \mathrm{P}_{2}$ binding, we performed cell fractionation experiments after incubation of cells with neomycin $(10 \mathrm{mM})$ known to bind, and thus to mask, $\mathrm{PI}(4,5) \mathrm{P}_{2}$ polar heads (Wang et al., 1984). It was recently shown that ERM proteins were mostly recovered in the soluble fraction after addition of neomycin in L cells (Yonemura et al., 2002). We used as a control the FERM domain of ezrin known to bind PI(4,5) $\mathrm{P}_{2}$ (Niggli et al., 1995; Barret et al., 2000) and, as expected, we found that neomycin treatment strongly reduced the association of the VSV-G epitope-tagged ezrin-FERM domain with cytoskeletal fraction (Fig. 9, A). First, we demonstrated that neomycin severely decreases the in vitro interaction of the PTPL1-FERM domain with PIPs on protein-lipid overlays (Fig. 9, B). We next observed that such a cell treatment also strongly reduced the presence of the wild-type PTPL1-FERM domain associated with the particulate fraction without having any effects on the repartition of KN1-2 mutant (Fig. 9, C). In the same manner, we showed that association of wt-PTPL1 with the cytoskeletal fraction was inhibited by $50 \%$ with neomycin addition (Fig. 8; 9, D). Moreover, subcellular distribution of KN1-2 or $\triangle$ FERM mutants was not affected by such a treatment (Fig. 9, D).

Those data strongly support the necessity of $\mathrm{PI}(4,5) \mathrm{P}_{2}$ binding for cytoskeletal association of PTPL1 through motifs comprised within its FERM domain. 


\section{$\underline{\text { Discussion }}$}

The knowledge of the PTP diversity has been addressed in the last 15 years after characterization of the first family member (Tonks et al., 1988). Most attention was originally drawn on the understanding of basic enzymatic function by searching for their specific substrates. The substrate-trapping approach, developed by Tonks's laboratory, constituted a powerful and valuable approach to identify many PTP substrates and discover pathways in which PTPs were involved (Garton et al., 1996; Flint et al., 1997). However, it soon becomes clear that the diversity of their functions could be a consequence of the regulation of their activity in a particular subcellular environment. In fact, the substrate specificity of cytosolic PTPs is thought to depend upon their intracellular targeting and in some cases on an intrinsic property carried by their catalytic domain (for review, Mauro and Dixon, 1994; Fischer, 1999; Tonks and Neel, 2001). Therefore, an important step in the understanding of a particular enzyme functions is the identification of its localization and regulatory mechanisms in different physiological contexts. In this study, we have defined human PTPL1 localization and demonstrated that phosphoinositides are playing a key role in the regulation of its membrane addressing.

PTPL1 belongs to the FERM domain-containing PTP superfamily comprising several mammalian enzymes PTPH1, PTPMEG, PTP36/Pez and PTPD1/PTP-RL10 as well as the recently identified worm PTP-FERM. In this report, we demonstrate by immunofluorescence using different tagged-constructs that the FERM domain of human PTPL1 is necessary and sufficient to target the enzyme at the apical face of cell membrane and in microvilli in HeLa epithelial cells. In accordance with our findings, PTP36, PTPH1, and PTPMEG have been reported to associate with the cell membrane (Ogata et al., 1999; Gjorloff-Wingren et al., 2000). The FERM domains of PTP-BL, the mouse homologue of PTPL1 and of the worm PTP-FERM, were shown to be sufficient for their submembranous distribution (Cuppen et al., 
1999; Uchida et al., 2002). Furthermore, FERM domain deletion from PTPH1, PTPMEG and PTP-FERM altered their intracellular targeting (Gjorloff-Wingren et al., 2000; Uchida et al., 2002). However, it does not seem to be a general feature for all family members since PTPD1 whose FERM domain is known to interact with KIF1C, a kinesin-like protein, was shown to be mainly localized in the cytoplasm (Dorner et al., 1998; Cuppen et al., 1999) whereas FERM domain of Pez was involved in the nuclear localization of this enzyme in HEK 293 cells at low density (Wadham et al., 2000).

The membraneous PTPL1 localization raises the question of its association with the cell cytoskeleton. By COS1 cell fractionation, we show that the FERM domain of PTPL1 is mainly recovered in the particulate fraction containing polymerized actin and vimentin. Moreover $35 \%$ of total full-length PTPL1 is also found to be associated with cytoskeletal fraction in a FERM domain-dependent manner. Having established that the FERM domain was involved in PTPL1 intracellular targeting and required for its association with cytoskeletal fraction, we next investigated how its localization could be regulated in order to identify physiological mechanisms controlling PTPL1 activity.

FERM domains of ERM proteins, composed of three subdomains (A, B and C), are known to bind transmembrane proteins like CD44, CD43, ICAM-1 and -3 and also PI(4,5) $\mathrm{P}_{2}$ (Hamada et al., 2000; for review, Bretscher, 1999). Ezrin binding to CD44 is increased by $\mathrm{PI}(4,5) \mathrm{P}_{2}$ in vitro (Hirao, 1996). Recently, it has been demonstrated that ezrin localization was altered by mutation of $\mathrm{PI}(4,5) \mathrm{P}_{2}$-binding motifs found in subdomains A and C (Barret et al., 2000). PTPL1 sequence alignment with ezrin points to two potential $\mathrm{PI}(4,5) \mathrm{P}_{2}$-binding sites in its FERM domain sequence in the same A and C subdomains (Fig. 3). The first site located at amino acids 641-647 corresponds to a KXXXKKK motif and resembles the consensus sequence in villin, $(\mathrm{K} / \mathrm{R}) \mathrm{XXXX}(\mathrm{K} / \mathrm{R})(\mathrm{K} / \mathrm{R})$. The second site, KKXXXXKKK, located at amino acids $824-832$, is similar but not identical to the first consensus sequence in ERM, 
$\mathrm{KKXXXXXX(K/R)K.} \mathrm{By} \mathrm{introducing} \mathrm{mutations} \mathrm{in} \mathrm{these} \mathrm{sites,} \mathrm{we} \mathrm{presently} \mathrm{found} \mathrm{that} \mathrm{lysine}$ to asparagine mutations of both sites $(\mathrm{K} 644,645,646,824,825,830,831,832 \mathrm{~N})$ altered PTPL1-FERM domain as well as full-length enzyme localization. Furthermore PTPL1 KN1-2 localization was similar to that observed with PTPL1 $\triangle$ FERM. In addition, we demonstrated that association of the FERM domain or full-length PTPL1 with cytoskeletal fraction was abrogated by mutation of both potential $\mathrm{PI}(4,5) \mathrm{P}_{2}$-binding motifs or FERM domain deletion. In fact, only $4 \%$ and $2 \%$ of PTPL1 KN1-2 and PTPL1 $\triangle$ FERM mutants remained respectively associated to the particulate fraction whereas $35 \%$ of total wild-type PTPL1 is found in that fraction. Our results altogether indicate that the two domains, located in regions 641-647 and 824-832, were responsible for PTPL1-FERM domain, and therefore for PTPL1, localization and association with cytoskeletal fraction.

By similarity to what has been described for ezrin (Barret et al., 2000), we hypothesized that these two domains do cooperate in $\mathrm{PI}(4,5) \mathrm{P}_{2}$ binding and we investigated the direct binding of PTPL1-FERM domain to PI(4,5) $\mathrm{P}_{2}$ in vitro. Our first attempt using FERM domains expressed in bacteria failed to give us results due the strong insolubility of this protein in absence of PIP2 (data not shown). However, labeled 6His-epitope tagged PTPL1-FERM domains obtained by coupled transcription/translation in vitro permitted to purify low amounts of tracer proteins which remain soluble at these concentrations and thus allow to probe nitrocellulose membrane containing immobilized PIPs. Using such protein-lipid overlays, we showed that the FERM domain of PTPL1 binds specifically 4' and/or 5' phosphorylated PIPs. KN2 mutation, and to a lesser extent KN1 mutation, were found to affect PIPs binding which is almost completely abolished by combination of both mutations (KN1-2). This result clearly demonstrates that the two suspected $\mathrm{PI}(4,5) \mathrm{P}_{2}$-binding sites are functional and cooperate for PIPs binding in vitro. The in vitro ERM FERM domain binding selectivity for PIPs is largely unknown. Among PIPs bound by the FERM domain of PTPL1, PI(4,5) $\mathrm{P}_{2}$ and PI(4)P are the 
most abundant in mammal cells (for review, Payrastre et al., 2001). PI(5)P, which represents with $\mathrm{PI}(3) \mathrm{P}$ around $0.25 \%$ of cellular phosphoinositides, is absent in certain cell lines, among them COS1 cells (Shisheva, 2001). As for higher 3' phosphorylated species known to be localized at the plasma membrane, as $\mathrm{PI}(3,4) \mathrm{P}_{2}$ and $\mathrm{PI}(3,4,5) \mathrm{P}_{3}$, their rates are generally low or absent and are strongly regulated by extracellular stimuli (Payrastre et al., 2001). Moreover, we showed that serum starvation of COS1 cells, known to reduce PI3-kinase activity and thus $\mathrm{PI}(3,4) \mathrm{P}_{2}$ and $\mathrm{PI}(3,4,5) \mathrm{P}_{3}$ levels, does not affect the localization and subcellular distribution of PTPL1 (data not shown). The nature of PIPs could affect binding affinity and selectivity. Our preliminary results on different protein-lipid overlays show that the binding of PTPL1-FERM domain to natural $\mathrm{PI}(4,5) \mathrm{P}_{2}$ is stronger than to synthetic $\mathrm{PI}(4,5) \mathrm{P}_{2}$ which displays shorter fatty acid chains than in natural $\mathrm{PI}(4,5) \mathrm{P}_{2}$ (data not shown). Data on PIPs binding, associated to the prevalent rate of $\mathrm{PI}(4,5) \mathrm{P}_{2}$ and its proper cellular localization, strongly favor $\mathrm{PI}(4,5) \mathrm{P}_{2}$ being responsible for PTPL1 localization and subcellular distribution.

Our data suggest that the association of PTPL1 with the particulate fraction depends on $\mathrm{PI}(4,5) \mathrm{P}_{2}$ binding and that at least the second motif within its FERM domain is essential. This first evidence was strongly reinforced by the demonstration that COS1 cell treatment with neomycin $(10 \mathrm{mM})$, a polycation which binds to the polar heads of $\mathrm{PI}(4,5) \mathrm{P}_{2}$ in the cell membrane and inhibits PTPL1-FERM domain interaction with $\mathrm{PI}(4,5) \mathrm{P}_{2}$ in overlay experiments, had similar effect on PTPL1 subcellular distribution than KN1-2 mutations. Indeed, the association of PTPL1 with the cytoskeletal fraction was reduced by $50 \%$ after neomycin treatment.

Our studies on PTPL1 bring the first argument for $\mathrm{PI}(4,5) \mathrm{P}_{2}$ binding being primarily responsible for PTP cellular targeting and subcellular distribution. It is interesting to note that among PTPs containing a FERM domain and known to localize at the plasma membrane 
PTPH1 and PTP-FERM also possess two potential PI(4,5) $\mathrm{P}_{2}$ binding sites situated in same regions to those of PTPL1 (Fig. 3). However, PTPL1 intracellular localization is much more limited than that of PI(4,5)P2, and the PH domains of PLC $\delta 1$ and oxysterol-binding protein (OSBP) known to specifically interact with $\mathrm{PI}(4,5) \mathrm{P}_{2}$ in vitro displayed different localizations when expressed in cells (for review, Balla et al., 2000). These data therefore suggest the existence of additional anchoring proteins which might contribute to membrane localization of $\mathrm{PI}(4,5) \mathrm{P}_{2}$ binding domains by restricting and stabilizing their recruitment into membrane specific compartments. We showed that the FERM domain as well as PTPL1 colocalized with F-actin at plasma membrane (cortical actin) and in microvilli. PTPMEG and PTP36 were reported to associate with cytoskeletal fraction ( $\mathrm{Gu}$ and Marjerus, 1996; Ogata et al., 1999; Aoyama et al., 1999). Furthermore, many proteins interacting with PDZ domains of human PTPL1 or mouse PTP-BL have been shown to be associated with cytoskeleton like PARG1, a Rho-GAP protein (Saras et al., 1997), ZRP-1, a zyxin-related protein or RIL, a LIM domaincontaining protein colocalazing with F-actin (Murthy et al., 1999; Cuppen et al., 1998) suggesting a potential role of PTPL1 in the dynamic of actin cytoskeleton. It has recently been shown that PTP-BL associates with midbody during the cell cycle and is involved in cytokinesis in HeLa cells (Herrmann et al., 2003). In this study, the FERM domain of PTP-BL was found to colocalize with F-actin at the contractile ring and to bind, directly or indirectly, F-actin in vitro. Moreover, the FERM domain of ezrin has been shown to bind G-actin as well as F-actin in vitro (Roy et al., 1997). Altogether those data suggest that the association of PTPL1 with the particulate fraction could be due to a direct or indirect binding to the actin cytoskeleton through its FERM domain. However, depletion of F-actin by treatment of COS1 cells with latrunculin A does not affect the subcellular distribution of PTPL1-FERM domain or full-length enzyme (data not shown). This discrepancy in PTPL1/PTP-BL localization could be explained by the PTPL1 gene structure which was recently described and revealed 
the existence of 47 exons raising many isoform possibilities (Ensembl gene ID, ENSG00000163629). Indeed, Herrmann et al. (2003) demonstrated differential localizations of some different isoforms of PTP-BL during the cell cycle.

In this report, we have discovered two main characteristics of human PTPL1 localization. First of all, we outlined the crucial role of the FERM domain for its submembraneous addressing. Second, we demonstrated that its association with the particulate fraction is a phenomenon driven by $\mathrm{PI}(4,5) \mathrm{P}_{2}$ interaction with specific binding motifs within this domain. Our major goal is now to reconcile this regulated subcellular localization with the potential functions of this enzyme in different cellular contexts. FERM domain deletion of PTPH1 was shown to block its inhibitory effect on T-cell antigen receptor signaling pointing that a proper localization is crucial for its physiologic effect (Han et al., 2000). In MCF7 human breast cancer cells, we previously showed that PTPL1 was involved in the inhibition of IGF-1 survival activity by blocking PI3-kinase (PI3-K) pathway and this inhibitory action occurs at early steps of IGF-I signaling (Freiss et al., 1998; Bompard et al., 2002). PTP-BL has recently been shown to localize in lipid rafts in mouse embryo and cortical neurons (Palmer et al., 2002). Futhermore, $\mathrm{PI}(4,5) \mathrm{P}_{2}$ has been demonstrated to accumulate within such lipid microdomains (Laux et al., 2000). The association of PTPL1 with the particulate fraction, not driven by F-actin binding, could thus result from its presence in lipid rafts since these domains are recovered in Triton X-100 insoluble fraction. In MCF7 cells, the IGF-I receptor (IGF-IR), as well as PI3-K, have been shown to transiently associate with lipid rafts after IGF-I stimulation (Manes et al., 1999). This transient localization could allow PTPL1, associated with lipid rafts, to inhibit the IGF-I survival pathway in human breast tumors where it could represent an interesting new therapeutic tool. 


\section{Acknowledgements}

The authors wish to thank Drs. Travo and Cau, from the Integrated Imaging Facility of the CRBM (Montpellier), and Nicole Lautrédou from the Imaging Service of the IGH (Montpellier) for their support. We are grateful to Dr. Joubert for fruitful discussions and for critical reading of the article.

This work was supported by Inserm and by the Association pour la Recherche sur le Cancer (ARC, grants 5405 and 5761). G.B was supported by a fellowship from the ARC. 


\section{$\underline{\text { References }}$}

Aoyama, K., Matsuda, T. and Aoki, N. (1999). Characterization of newly identified four isoforms for a putative cytosolic protein tyrosine phosphatase PTP36. Biochem. Biophys. Res. Commun. 266, 523-531.

Balla, T., Bondeva, T. and Varnai, P. (2000). How accurately can we image inositol in living cells? Trends Pharmacol. Sci. 21, 238-241.

Banville, D., Ahmad, S., Stocco, R. and Shen, S. H. (1994). A novel protein-tyrosine phosphatase with homology to both the cytoskeletal proteins of the band 4.1 family and junction-associated guanylate kinases [published erratum appears in J Biol Chem 1995 Apr 28;270(17):10359]. J. Biol. Chem. 269, 22320-22327.

Barret, C., Roy, C., Montcourrier, P., Mangeat, P. and Niggli, V. (2000). Mutagenesis of the phosphatidylinositol 4,5-bisphosphate ( $\mathrm{PIP}(2))$ binding site in the $\mathrm{NH}(2)$-terminal domain of ezrin correlates with its altered cellular distribution. J. Cell Biol. 151, 1067-1080.

Bompard, G., Prebois, C., Puech, C., Vignon, F. and Freiss, G. (2002). Protein-tyrosine phosphatase PTPL1/FAP-1 triggers apoptosis in human breast cancer cells. J. Biol. Chem. 277, 47861-47869.

Bretscher, A. (1999). Regulation of cortical structure by the ezrin-radixin-moesin protein family. Curr. Opin. Cell Biol. 11, 109-116.

Chishti, A. H., Kim, A. C., Marfatia, S. M., Lutchman, M., Hanspal, M., Jindal, H., Liu, S. C., Low, P. S., Rouleau, G. A., Mohandas N. et al. (1998). The FERM domain: a unique module involved in the linkage of cytoplasmic proteins to the membrane. Trends Biochem. Sci. 23, 281-282. 
Cuppen, E., Gerrits, H., Pepers, B., Wieringa, B. and Hendriks, W. (1998). PDZ motifs in PTP-BL and RIL bind to internal protein segments in the LIM domain protein RIL. Mol. Biol. Cell 9, 671-683.

Cuppen, E., Wijers, M., Schepens, J., Fransen, J., Wieringa, B. and Hendriks, W. (1999). A FERM domain governs apical confinement of PTP-BL in epithelial cells. J. Cell Sci. 112, 3299-3308.

Dorner, C., Ciossek, T., Muller, S., Moller, P. H., Ullrich A. and Lammers, R. (1998). Characterization of KIF1C, a new kinesin-like protein involved in vesicle transport from the Golgi apparatus to the endoplasmic reticulum. J. Biol. Chem. 273, 20267-20275.

Fischer, E. H. (1999). Cell signaling by protein tyrosine phosphorylation. Adv. Enzyme Regul. 39, 359-369.

Flint, A. J., Tiganis, T., Barford, D. and Tonks, N. K. (1997). Development of "substratetrapping" mutants to identify physiological substrates of protein tyrosine phosphatases. Proc. Natl. Acad. Sci.U.S.A. 94, 1680-1685.

Freiss, G. and Vignon, F. (1994). Antiestrogens increase protein tyrosine phosphatase activity in human breast cancer cells. Mol. Endocrinol. 8, 1389-1396.

Freiss, G., Puech, C. and Vignon, F. (1998). Extinction of insulin-like growth factor-I mitogenic signaling by antiestrogen-stimulated Fas-associated protein tyrosine phosphatase-1 in human breast cancer cells. Mol. Endocrinol. 12, 568-579.

Garton, A. J., Flint, A. J. and Tonks, N. K. (1996). Identification of p130(cas) as a substrate for the cytosolic protein tyrosine phosphatase PTP-PEST. Mol. Cell. Biol. 16, 6408-6418. 
Gjorloff-Wingren, A., Saxena, M., Han, S., Wang, X., Alonso, A., Renedo, M., Oh, P., Williams, S., Schnitzer, J. and Mustelin, T. (2000). Subcellular localization of intracellular protein tyrosine phosphatases in T cells. Eur. J. Immunol. 30, 2412-2421.

Gu, M. and Majerus, P. W. (1996). The properties of the protein tyrosine phosphatase PTPMEG. J. Biol. Chem. 271, 27751-27759.

Gu, M. X., York, J. D., Warshawsky, I. and Majerus, P. W. (1991). Identification, cloning, and expression of a cytosolic megakaryocyte protein-tyrosine-phosphatase with sequence homology to cytoskeletal protein 4.1. Proc. Natl. Acad. Sci. U.S.A 88, 5867-5871.

Hamada, K., Shimizu, T., Matsui, T., Tsukita, S. and Hakoshima, T. (2000). Structural basis of the membrane-targeting and unmasking mechanisms of the radixin FERM domain. EMBO J. 19, 4449-4462.

Han, S., Williams, S. and Mustelin, T. (2000). Cytoskeletal protein tyrosine phosphatase PTPH1 reduces T cell antigen receptor signaling. Eur. J. Immunol. 30, 1318-1325.

Herrmann, L., Dittmar, T. and Erdmann, K. S. (2003). The protein tyrosine phosphatase PTP-BL associates with midbody and is involved in the regulation of cytokinesis. Mol. Biol. Cell 14, 230-240.

Higashitsuji, H., Arii, S., Furutani, M., Imamura, M., Kaneko, Y., Takenawa, J., Nakayama, H. and Fujita, J. (1995). Enhanced expression of multiple protein tyrosine phosphatases in the regenerating mouse liver: isolation of PTP-RL10, a novel cytoplasmictype phosphatase with sequence homology to cytoskeletal protein 4.1. Oncogene 10, 407414. 
Hirao, M., Sato, N., Kondo, T., Yonemura, S., Monden, M., Sasaki, T., Takai, Y., Tsukita, S. and Tsukita, S. (1996). Regulation mechanism of ERM (ezrin/radixin/moesin) protein/plasma membrane association: possible involvement of phosphatidylinositol turnover and Rho-dependent signaling pathway. J. Cell Biol. 135, 37-51.

Hunter, T. (1997). Oncoprotein networks. Cell 88, 333-346.

Laux, T., Fukami, K., Thelen, M., Golub, T., Frey, D. and Caroni, P. (2000). GAP43, MARKS, and CAP23 modulate $\mathrm{PI}(4,5) \mathrm{P}_{2}$ at plasmalemmal rafts, and regulate cell cortex actin dynamics through a common mechanism. J. Cell Biol. 149, 1455-1471.

Maekawa, K., Imagawa, N., Nagamatsu, M. and Harada, S. (1994). Molecular cloning of a novel protein-tyrosine phosphatase containing a membrane-binding domain and GLGF repeats. FEBS Lett. 337, 200-206.

Manes, S., Mira, E., Gomez-Mouton, C., Lacalle, R. A., Keller, P., Labrador, J. P. and Martinez-A, C. (1999). Membrane raft microdomains mediate front-rear polarity in migrating cells. EMBO J. 18, 6211-6220.

Mangeat, P., Roy, C. and Martin, M. (1999). ERM proteins in cell adhesion and membrane dynamics. Trends Cell Biol. 9, 187-192.

Martin, M., Roy, C., Montcourrier, P., Sahuquet, A., and Mangeat, P. (1997). Three determinants in ezrin are responsible for cell extension activity. Mol. Biol. Cell 8, 15431557.

Mauro, L. J. and Dixon, J. E. (1994). 'Zip codes' direct intracellular protein tyrosine phosphatases to the correct cellular 'address'. Trends Biochem. Sci. 19, 151-155. 
Moller, N. P., Moller, K. B., Lammers, R., Kharitonenkov, A., Sures, I. and Ullrich, A. (1994). Src kinase associates with a member of a distinct subfamily of protein-tyrosine phosphatases containing an ezrin-like domain. Proc. Natl. Acad. Sci. U.S.A 91, 7477-7481.

Murthy, K. K., Clark, K., Fortin, Y., Shen, S. H. and Banville, D. (1999). ZRP-1, a zyxinrelated protein, interacts with the second PDZ domain of the cytosolic protein tyrosine phosphatase hPTP1E. J. Biol. Chem. 274, 20679-20687.

Neel, B. G. and Tonks, N. K. (1997). Protein tyrosine phosphatases in signal transduction. Curr. Opin. Cell Biol. 9, 193-204.

Niggli, V., Andreoli, C., Roy, C. and Mangeat, P. (1995). Identification of a phosphatidylinositol-4,5-bisphosphate-binding domain in the N-terminal region of ezrin. FEBS Lett. 376 , 172-176.

Ogata, M., Takada, T., Mori, Y., Oh-hora, M., Uchida, Y., Kosugi, A., Miyake, K. and Hamaoka, T. (1999). Effects of Overexpression of PTP36, a Putative Protein Tyrosine Phosphatase, on Cell Adhesion, Cell Growth, and Cytoskeletons in HeLa Cells. J. Biol. Chem. 274, 12905-12909.

Palmer, A., Zimmer, M., Erdmann, K. S., Eulenburg, V., Porthin, A., Heumann, R., Deutsch, U. and Klein, R. (2002). EphrinB phosphorylation and reverse signaling: regulation by src kinases and PTP-BL phosphatase. Mol. Cell 9, 724-737.

Payrastre, B., Missy, K., Giuriato, S., Bodin, S., Plantavid, M. and Gratacap, M. P. (2001). Phosphoinositides: key players in cell signalling, in time and space. Cell. Signal. 13, 377-387. 
Roy, C., Martin, M. and Mangeat. P. (1997). A dual involvement of the amino-terminal domain of ezrin in F- and G-actin binding. J. Biol. Chem. 272, 20088-20095.

Saras, J., Claesson-Welsh, L., Heldin, C. H. and Gonez, L. J. (1994). Cloning and characterization of PTPL1, a protein tyrosine phosphatase with similarities to cytoskeletalassociated proteins. J. Biol. Chem. 269, 24082-24089.

Saras, J., Franzen, P., Aspenstrom, P., Hellman, U., Gonez, L. J. and Heldin, C. H. (1997). A novel GTPase-activating protein for Rho interacts with a PDZ domain of the protein-tyrosine phosphatase PTPL1. J. Biol. Chem. 272, 24333-24338.

Sato, T., Irie, S., Kitada, S. and Reed, J. C. (1995). FAP-1: a protein tyrosine phosphatase that associates with Fas. Science 268, 411-415.

Sawada, M., Ogata, M., Fujino, Y. and Hamaoka, T. (1994). cDNA cloning of a novel protein tyrosine phosphatase with homology to cytoskeletal protein 4.1 and its expression in T-lineage cells. Biochem. Biophys. Res. Commun. 203, 479-484.

Shisheva, A. (2001). PIKfyve: the road to PtdIns 5-P and PtdIns 3,5- $\mathrm{P}_{2}$. Cell Biol. Int. 25, 1201-1206.

Smith, A. L., Mitchell, P. J., Shipley, J., Gusterson, B. A., Rogers, M. V. and Crompton, M. R. (1995). Pez: a novel human cDNA encoding protein tyrosine phosphatase- and ezrinlike domains. Biochem. Biophys. Res. Commun. 209, 959-965.

Tonks, N. K., Diltz, C. D. and Fischer, E. H. (1988). Purification of the major proteintyrosine-phosphatases of human placenta. J. Biol. Chem. 263, 6722-6730.

Tonks, N. K. and Neel, B. G. (2001). Combinatorial control of the specificity of protein tyrosine phosphatases. Curr. Opin. Cell Biol. 13, 182-195. 
Uchida, Y., Ogata, M., Mori, Y., Oh-hora, M., Hatano, N. and Hamaoka, T. (2002). Localization of PTP-FERM in nerve processes through its FERM domain. Biochem. Biophys. Res. Commun. 292, 13-19.

Wadham, C., Gamble, J. R., Vadas, M. A. and Khew-Goodall, Y. (2000). Translocation of protein tyrosine phosphatase Pez/PTPD2/PTP36 to the nucleus is associated with induction of cell proliferation. J. Cell Sci. 113 ( Pt 17), 3117-3123.

Wallace, M. J., Fladd, C., Batt, J. and Rotin, D. (1998). The second catalytic domain of protein tyrosine phosphatase delta (PTP delta) binds to and inhibits the first catalytic domain of PTP sigma. Mol. Cell. Biol. 18, 2608-2616.

Wang, B.M., Weiner, N.D., Takada, A. and Schacht, J. (1984). Characterization of aminoglycoside-lipid interactions and development of a refined model for ototoxicity testing. Biochem. Parmacol. 33, 3257-3262.

Yang, Q. and Tonks, N. K. (1991). Isolation of a cDNA clone encoding a human proteintyrosine phosphatase with homology to the cytoskeletal-associated proteins band 4.1, ezrin, and talin. Proc. Natl. Acad. Sci. U.S.A 88, 5949-5953.

Yonemura, S., Matsui, T., Tsukita, S. and Tsukita, S. (2002). Rho-dependent and -independent activation mechanisms of ezrin/radixin/moesin proteins: an essential role for polyphosphoinositides in vivo. J.Cell Sci. 115, 2569-2580.

Zhang, Z. Y. (2001). Protein tyrosine phosphatases: prospects for therapeutics. Curr. Opin. Chem. Biol. 5, 416-423. 


\section{Figure legends}

Figure 1: (A) Schematic representation of the PTPL1 expression constructs used. The modular protein domains are indicated in square. Protein segments produced by transient expression are indicated within parentheses, numbers corresponding to the first and last amino acids positions according to PTPL1 sequence (EMBL accession X80289). (B) Expression of PTPL1-FERM constructs. COS1 cells were transiently transfected with HA-epitope tagged PTPL1-FERM constructs: wt (lane1), KN1 (lane 2), KN2 (lane 3) or KN1-2 (lane 4) mutants. Cells were lysed in SDS sample buffer, fractionated on a 12.5\% SDS-PAGE and expression was revealed after Western-blot with anti-HA Ab. (C) Expression of full-length PTPL1 constructions. COS1 cells were transiently transfected with HA-epitope tagged PTPL1 constructs: wt (lane1), KN1-2 (lane 2) or $\triangle$ FERM mutants. Expression was revealed as described above after a 7\% SDS-PAGE. * indicated non specific band revealed with anti-HA antibody.

Figure 2: The FERM domain of PTPL1 is necessary and sufficient for its apical localization and microvilli association in HeLa cells. Full length PTPL1 (A-C), PTPL1-FERM domain (DF) and PTPL1 $\triangle$ FERM (G-I) with HA-epitope tags were transiently expressed in HeLa epithelial cells (see Fig. 1). Cells were treated for indirect FITC localization of PTPL1 constructs with anti-HA antibody (left) and F-actin with TRITC-coupled phalloidin (middle). Images represent merges of horizontal confocal sections. Vertical xz sections at the level of the dotted lines are presented under each figure. Dual localizations of PTPL1 constructs and actin are merged in colour, yellow represents colocalization. Full length PTPL1 is located at the apical face of cell membrane (A) and colocalized with F-actin at the level of dorsal microvilli (C, arrow) and lateral cell membranes (C). The PTPL1-FERM domain displayed 
the same subcellular localization (F, arrow) than the full length enzyme. Cell extensions were also observed (D, arrowhead). On the contrary, PTPL1 $\triangle$ FERM was found mainly cytosolic and enriched around the nucleus (G, arrow). Bars, $20 \mu \mathrm{m}$.

Figure 3: Alignment of FERM domain sequences from PTPs and ezrin. The aligned FERM domains are human PTPH1 (hPTPH1), C. elegans PTP-FERM (PTP-FERM), human PTPL1 (hPTPL1) and human ezrin (hEzrin). Conserved amino acid residues from at least two sequences are highlighted in black when identical or in grey when encompassing to the same family. Bold bars represent localization of the two $\mathrm{PI}(4,5) \mathrm{P}_{2}$-binding motifs identified in ezrin. Subdomains A, B and C of ezrin are indicated by thin bars.

Figure 4: The localization of the PTPL1-FERM domain is altered by mutation of the two potential $\mathrm{PI}(4,5) \mathrm{P}_{2}$-binding sites in $\mathrm{COS} 1$ cells. $\mathrm{COS} 1$ cells were transfected with HA-epitope tagged wt-FERM domain (A), KN1-FERM mutant (B, K645, 646, 647N), KN2-FERM mutant $(\mathrm{C}, \mathrm{K} 824,825,830,831,832 \mathrm{~N})$ and KN1-2-FERM mutant (D, combination of KN1 and KN2 mutations) and treated for immunolocalization of HA-epitope tagged constructs as described in Figure 2. Transfected wild-type FERM domain, KN1 and KN2 mutants were associated with plasma membrane, dorsal microvilli and induced membrane extensions (A, B and C, arrowheads). KN1-2 mutant of FERM domain was mainly localized in cytoplasm (D, arrowhead). Bars, $10 \mu \mathrm{m}$.

Figure 5: Mutation of both potential $\mathrm{PI}(4,5) \mathrm{P}_{2}$-binding motifs altered subcellular localization of FERM domain as well as full-length PTPL1 in HeLa cells. HA-epitope tagged PTPL1FERM domain (A-C) or full length PTPL1 (D-F) carrying KN1-2 mutations were transiently expressed in HeLa cells. Cells were treated for indirect immunofluorescence of PTPL1 
constructs (A and D) and F-actin (B and E) as described in Figure 2. Dual localizations are merged in colour $(\mathrm{C}$ and $\mathrm{F})$. Images represent merges of horizontal confocal planes. Both constructs displayed a similar cytosolic localization enriched around nucleus (A, arrow; E, arrow). Bars, $20 \mu \mathrm{m}$.

Figure 6: PTPL1-FERM interaction with PIPs. ${ }^{35}$ S-labeled PTPL1-FERM domain (wt) and its $\mathrm{KN} 1, \mathrm{KN} 2, \mathrm{KN} 1-2$ mutants were produced by in vitro transcription-coupled translation system. (A) 4,000 cpm of each labeled recombinant protein were analysed on a $10 \%$ SDS/PAGE and submitted to autoradiography. (B) 40,000 cpm of each labeled protein were incubated with PIP-Strips (Echelon Biosciences Inc.) plotted with 100 pmol of PtdIns, PtdIns(3)P [PI(3)P], PtdIns(4)P [PI(4)P], PtdIns(5)P [PI(5)P], PtdIns(3,4) $\mathrm{P}_{2}$ [PI(3,4)P2], $\operatorname{PtdIns}(3,5) \mathrm{P}_{2} \quad[\mathrm{PI}(3,5) \mathrm{P} 2], \quad \operatorname{PtdIns}(4,5) \mathrm{P}_{2} \quad[\mathrm{PI}(4,5) \mathrm{P} 2], \quad \operatorname{PtdIns}(3,4,5) \mathrm{P}_{3} \quad[\mathrm{PI}(3,4,5) \mathrm{P} 3]$, phosphatidylethanolamine (PE), phosphatidylcholine (PC), phosphatidylserine (PS), lysophosphatidic acid (LPA), lysophosphacholine (LPC), sphingosine-1-phosphate (S1P) and phosphatic acid (PA). After 3 washes, PIP-strips were submitted to autoradiography.

Figure 7: Subcellular localization of transfected mutated or wild-type PTPL1-FERM domain or full-length PTPL1. Transfected COS1 cells were fractionated as described in Materials and Methods and the resulting fractions were analysed on a 10\% SDS/PAGE and immunoblotted. F-actin and intermediate filaments containing vimentin were recovered in the particulate fraction (P: pellet), whereas G-actin and depolymerised tubulin were recovered in the soluble fraction (S: supernatant). The equal amount of proteins loaded in each fraction was checked by Coomassie blue staining. (A) Transfection of FERM domains. Transfected HA-epitope tagged wild-type FERM domain was found to be strongly associated with the $\mathrm{P}$ fraction as detected after immunoblotting with anti-HA mAb. KN1-FERM mutant displayed an 
equivalent distribution. KN2- and KN1-2-FERM mutants were only detected in the S fraction. (B) Transfection of full-length PTPL1. Transfected HA-epitope tagged PTPL1 was mainly recovered in the $\mathrm{S}$ fraction and significantly in the P fraction. KN1-2 or $\triangle$ FERM mutant were only detected in the $\mathrm{S}$ fraction. To ensure proper fractionation, membranes were immunoblotted with anti-actin $\mathrm{Ab}$, anti-vimentin $\mathrm{mAb}$ and anti-tubulin $\mathrm{mAb}$.

Figure 8: Effects of FERM mutations (KN1-2 and $\triangle \mathrm{FERM})$ and neomycin treatment on the association of PTPL1 with the cytoskeletal fraction. The amount of PTPL1 and its mutants with the particulate (P) fraction after COS1 cell fractionation (see Fig.7, 9) treated or not with neomycin was quantified after densitometry of several immunoblots. White and black columns represent the percentage of PTPL1 associated with the cytoskeletal fraction in the absence or presence of neomycin treatment respectively. Association of wild-type PTPL1 with the cytoskeletal fraction was significantly different after neomycin treatment $(\mathrm{P}<0.005$, Student's $t$-test, $\mathrm{n}=10)$ and almost unchanged for KN1-2 and $\triangle$ FERM mutants $(\mathrm{P}=0.33$ and $\mathrm{P}=0.42$ respectively, Student's $t$-test, $\mathrm{n}=9$ ). Mean $+/-\mathrm{SD}$ of $n$ experiments (numbers in parentheses).

Figure 9: Effect of neomycin treatment on subcellular distribution of PTPL1. (A) Transfection of ezrin-FERM domain. COS1 cells were transfected with VSV-G-epitope tagged N-terminal part of ezrin (FERM domain, 1-310 aa) and were fractionated as described in Fig. 7 after treatment or not with $10 \mathrm{mM}$ neomycin for 24 hours. Association of ezrin-FERM domain with particulate fraction was strongly inhibited by such cell treatment. (B) ${ }^{35}$ S-labeled PTPL1FERM domain was incubated in TBST 3\% BSA with PIP-Strips (Echelon Biosciences Inc.) pre-saturated with TBST 3\% BSA [C] or TBST 3\% BSA containing 1mM neomycin [Neo]. (C) Transfection of PTPL1-FERM domains. COS1 cells were transfected with either HA- 
epitope tagged wild-type FERM domain or KN1-2-FERM domain, treated or not with neomycin as described above. Similarly to ezrin-FERM domain, association of wild-type FERM domain with P fraction was impaired by neomycin treatment. At the opposite KN1-2FERM mutant distribution was not affected by such a treatment. (D) Transfection of fulllength PTPL1. Similarly, COS1 cells were transfected with HA-epitope tagged wild-type PTPL1, PTPL1 KN1-2 or PTPL1 $\triangle$ FERM and fractionated after neomycin treatment. Neomycin treatment strongly inhibited the association of wild-type PTPL1 with the P fraction. Moreover, KN1-2 or $\triangle \mathrm{FERM}$ mutant distributions were not affected. 